\title{
The Role of 4DCT in the Evaluation of Patients with Bio-Prosthetic Valve Thrombosis
}

\author{
Parivash Badar*, Deepika Gopalakrishnan, Ambarish Gopal and Alicia Avila \\ Baylor Scott \& White The Heart Hospital Plano, Plano TX
}

*Corresponding author: Parivash Badar, Baylor Scott \& White The Heart Hospital

Plano, Plano TX, USA.

Received Date: May 10, 2019

Published Date: May 22, 2019

\section{Mini Review}

Valvular heart disease affects $>100$ million patients worldwide out of which 200,000 cases of aortic valve replacements are performed annually and the numbers are predicted to rise further due to the aging population and a subsequent increase in degenerative valve disease. Based on an analysis of the Society of Thoracic Surgeons National Database; between 1997 and 2006, Bioprosthetic tissue valve replacement gained prominence causing a massive shift from mechanical to bioprosthetic valve replacements. The use of mechanical valves decreased to $20.5 \%$, whereas the use of bioprosthetic valves increased to $78.4 \%[1,2]$ The transition from mechanical to bioprosthetic valve was partially attributed to the fact that, younger individuals refuse long-term anticoagulation and elderly patients are at a higher bleeding risk [1].

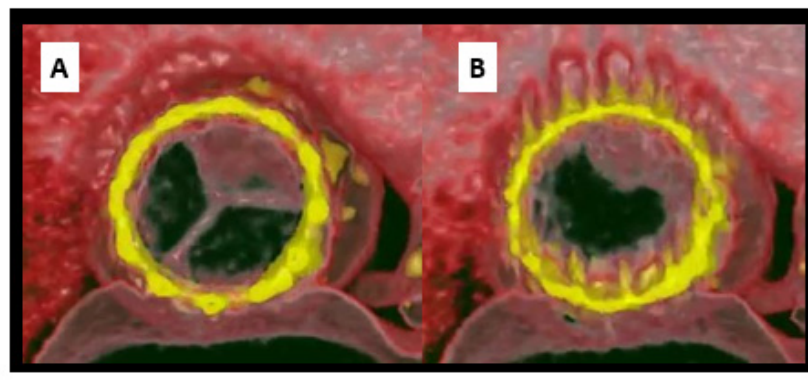

Figure 1A: Short axis view with 4DCT of the Aortic Valve Replacement showing clot in one leaflet during diastole.

Figure 1B: Short axis view with 4DCT of the Aortic Valve Replacement showing clot restricting leaflet excursion during systole.

Historically, it was regarded that chronic anti-coagulation and monitoring could be deferred in patients receiving bioprosthetic tissue valves. However, recent data shows bioprosthetic tissue valves could also develop thrombosis, particularly since the utilization of 4D CT angiography (4DCT) in practice. Most cases of valve thrombosis were diagnosed at a median of 6 months post-procedure, with patients presenting with Heart-Failure like symptoms i.e.; progressive dyspnea with exertion, low left ventricular ejection fractions, orthopnea, paroxysmal nocturnal dyspnea, and patients demonstrating an increased requirement of diuretics [3-6] Figure 1. Other likely predictors of valve thrombosis include paroxysmal atrial fibrillation, sub-therapeutic INR in patient who were anticoagulated with vitamin $\mathrm{K}$ antagonists, increased body mass index and lack of anti-coagulation [1,7]. Valve thrombosis may be associated with smaller valves $<23 \mathrm{~mm}$ or valve in valve procedures $[3,4]$.

Echocardiographic findings of significant increase in transvalvular gradient [5] in addition to leaflet thickening and abnormal cusp mobility serves as independent predictors of valve thrombosis. 4DCT, with its superior spatial resolution, is superior to echocardiography in analyzing surgical aortic valve replacement (SAVR)/Trans-catheter Aortic Valve Replacement (TAVR) leaflets' morphology and excursion [3-6,7,8,9] (Figure 2A\&2B).

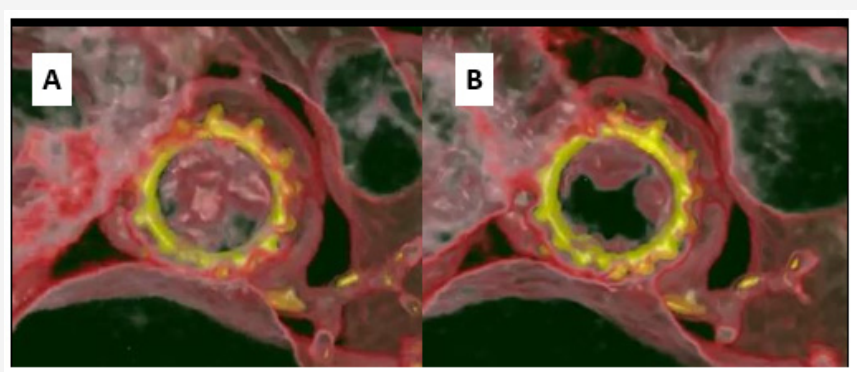

Figure 2A: 4DCT from another patient, with valve thrombosis, showing clot in two leaflets during diastole.

Figure 2B: 4DCT from another patient, with valve thrombosis, showing restriction of excursion of the opening of two leaflets during systole.

The latest generation CT scanners with their superior temporal and spatial resolution are now being increasingly utilized to further analyze valve replacements in an accurate manner. They are also 
able to identify degenerative disease of the bioprosthetic valve replacements by picking up leaflet calcification as well. 4DCT was thus is considered not only superior in terms of its imaging capabilities but it's also considered a sensitive marker for early calcification suggestive of bioprosthesic stenosis in the making and hence has a lower threshold for identifying such cases $[3,4,7,10$ 13]. In our clinical practice we treat symptomatic patients with Warfarin when indicated and follow up and monitor them with echocardiography and 4DCT. Patients who receive prompt anticoagulation and monitoring are often resolved of further symptoms and see better outcomes.

\section{Conclusion}

4DCT with its advanced temporal and spatial imaging capability is an imaging modality that has helped shape clinical practice in patients with bioprosthetic valve thrombosis and is effective in clinical decision making for anti-coagulation therapy $[3,4,7,10$ 13]. An increased awareness of symptoms and echocardiographic parameters is necessary when managing such patients. We look forward to more studies in the future that will put light on this topic [14-18].

\section{Acknowledgement}

None.

\section{Conflict of Interest}

No conflict of interest.

\section{References}

1. Bax Jeroen J, Victoria Delgado (2017) Bioprosthetic Heart Valves, Thrombosis, Anticoagulation, and Imaging Surveillance. JACC Cardiovascular Interventions 10(4): 388-390.

2. Brown JM, O Brien SM, Wu C, Sikora JA, Griffith BP, et al. (2009) Isolated Aortic Valve Replacement in North America Comprising 108,687 Patients in 10 Years: Changes in Risks, Valve Types, and Outcomes in the Society of Thoracic Surgeons National Database. The Journal of Thoracic and Cardiovascular Surgery US National Library of Medicine 137(1): 8290.

3. Basra SS, Gopal A, Hebeler KR, Baumgarten H, Anderson A, et al. (2018) Clinical Leaflet Thrombosis in Transcatheter and Surgical Bioprosthetic Aortic Valves by Four-Dimensional Computed Tomography. The Annals of Thoracic Surgery US National Library of Medicine 106(6): 1716-1725.

4. Wilson, Ryan (2019) Transcatheter Aortic and Mitral Valve Thrombosis. ClinicalKey.

5. De Marchena E, Mesa J, Pomenti S, Marin Y Kall C, Marincic X, et al. (2015) Thrombus Formation Following Transcatheter Aortic Valve
Replacement. JACC Cardiovascular Interventions US National Library of Medicine 8(5): 728-739.

6. Córdoba Soriano JG, Puri R, Amat Santos I, Ribeiro HB, Abdul Jawad Altisent 0 , et al. (2015) Valve Thrombosis Following Transcatheter Aortic Valve Implantation: a Systematic Review. Revista Espanola De Cardiologia (English Ed.) US National Library of Medicine 68(3): 198204.

7. Egbe AC, Pislaru SV, Pellikka PA, Poterucha JT, Schaff HV, et al. (2015) Bioprosthetic Valve Thrombosis Versus Structural Failure. JACC Journal of the American College of Cardiology 66(21): 2285-2294

8. Brown JM, O Brien SM, Wu C, Sikora JA, Griffith BP, et al. (2009) Isolated Aortic Valve Replacement in North America Comprising 108,687 Patients in 10 Years: Changes in Risks, Valve Types, and Outcomes in the Society of Thoracic Surgeons National Database. The Journal of Thoracic Surgery 137(1): 82-90.

9. Egbe AC, Connolly HM, Pellikka PA, Schaff HV, Hanna R, et al. (2017) Outcomes of Warfarin Therapy for Bioprosthetic Valve Thrombosis of Surgically Implanted Valves: A Prospective Study. JACC Cardiovascular Interventions US National Library of Medicine 10(4): 379-387.

10.(2019) Approach to Suspected Left-Sided Bioprosthetic Valve Obstruction and/or Thrombosis.

11. Pislaru SV, Hussain I, Pellikka PA, Maleszewski JJ, Hanna RD, et al. (2015) Misconceptions, diagnostic challenges and treatment opportunities in bioprosthetic valve thrombosis: lessons from a case series. Eur J Cardiothorac Surg 47: 725-732.

12. Latib A, Naganuma T, Abdel Wahab M, Danenberg H, Cota L, et al. (2015) Treatment and Clinical Outcomes of Transcatheter Heart Valve Thrombosis. Circulation Cardiovascular Interventions US National Library of Medicine 8(4)

13. Gopal A, Ribeiro N, Squiers JJ, Holper EM, Black M, et al. (2017) Pathologic Confirmation of Valve Thrombosis Detected by Four-Dimensional Computed Tomography Following Valve-in-Valve Transcatheter Aortic Valve Replacement. Global Cardiology Science \& Practice, Magdi Yacoub Heart Foundation (2):15

14. Tay EL, Gurvitch R, Wijeysinghe N, Nietlispach F, Wood D, et al. (2011) Valve Thrombosis after Transcatheter Heart Valve Implantation. Eurointervention 7(1): 170-171.

15. Fan J, Lipatov K, Lane W, Mixon T (2018) Management of Bioprosthetic Cardiac Valve Thrombosis. Proceedings (Baylor University Medical Center) Taylor \& Francis 31(4): 496-498.

16. Rishi Puri, Vincent Auffret, Josep Rodés Cabau (2017) Bioprosthetic Valve Thrombosis. Journal of the American College of Cardiology US National Library of Medicine 69(17): 2193-2211.

17. Raj R Makkar, Gregory Fontana, Hasan Jilaihawi, Tarun Chakravarty, Klaus F Kofoed, et al. (2015) Possible Subclinical Leaflet Thrombosis in Bioprosthetic Aortic Valves. The New England Journal of Medicine US National Library of Medicine 373: 2015-2024.

18. Puri R, Auffret V, Rodés Cabau J (2017) Bioprosthetic valve thrombosis. J Am Coll Cardiol 69(17): 2193-2211. 